\title{
A naturalistic observational study of patients with bipolar affective disorder from two tertiary care hospitals in Sri Lanka
}

P Kandapola Arachchige, KMIWM Senevirathne, VP Eranga, PLN Fernando, MUPK Peris, KALA Kuruppuarachchi, SS Williams

\section{Abstract \\ Introduction}

Bipolar affective disorder (BAD) is a lifelong condition with a variable course. The objective of this study was to conduct a naturalistic observation of the course of this disorder among patients attending two general hospital psychiatric clinics in Sri Lanka.

\section{Methods}

Adult patients with a diagnosis of BAD for more than 12 months were included in this cross sectional descriptive study. Those who were suffering from acute exacerbations, had schizoaffective disorder or alcohol or drug misuse were excluded. Data was collected from the patients, caregivers and clinic records using a pre-tested interviewer administered questionnaire. Outcomes of interests were socio demographic characteristics, duration of the illness, number of relapses, treatment adherence, suicidality and level of functioning.

\section{Results}

Of the 350 patients studied, majority $(55.7 \%)$ were females. Most (54.9\%) had their first relapse within 2-5 years from onset of their illness. Of them $27.7 \%$ experienced their first relapse within one year. The first relapse occurred within 6-10 years of onset of illness in $8.9 \%$, after 10 years in $6.3 \%$ and after 20 years in $2.3 \%$. One third $(33.4 \%)$ of patients had no second relapse. However, $38.3 \%$ relapsed for the second time within 2-5 years of diagnosing their illness. A minority (2.4\%) had a second relapse within the first year and another minority (2.6\%) after 20 years.

The likelihood of a relapse for each patient per year was 0.644 in the first 5 years, 0.405 in the next 5-10 years, 0.38 in the next $10-15$ years and 0.30 after 15 years.

\section{Conclusions}

Despite the relapsing nature of $\mathrm{BAD}$, predicting the course of the disorder in individual patients remains elusive. The trend was suggestive of fewer episodes over time, possibly mediated by better medication compliance and positive attitudes to treatment.

Keywords: bipolar affective disorder, relapse, Sri Lanka

SL J Psychiatry 2017; 8(2): 4-8

\section{Introduction}

Bipolar affective disorder (BAD) is a recurrent mood disorder with a variable prognosis (1). It is characterised by repeated (at least two) episodes of mood disturbance, which is associated with impairment of the patient's activity level. This disturbance consists of episodes of depression, mania or hypomania and mixed affective states (2). In BAD, mood shifts vary widely in their pattern, severity and frequency $(1,3,4)$. Some persons with BAD are more prone to either mania or depression, while others alternate equally between the two types of episodes (1). In some individuals, depression and mania may rapidly alternate and this is classified as rapid cycling (2).

The lifetime risk of BAD is between $0.3 \%-1.5 \%(5)$. It is equally prevalent in both males and females and is seen across all cultures and ethnic groups (6). Family, adoption and twin studies have established that the heritability of
BAD is high $(7,8)$. Environmental factors may however have a significant role in the development and course of BAD (9). There is consistent evidence from prospective studies that recent life events and interpersonal relationship issues contribute to the onset and relapse of BAD (10). Substance use, personality factors and physical causes may also precipitate episodes of the illness (11). Poor treatment adherence is another common reason for relapse (12).

BAD carries a high risk of suicide, especially during depressive episodes. One out of three people with BAD report past suicidal attempts or commit suicide, and this rate is 10-20 times higher than in the general population $(13,14)$. One study reports that between $25 \%$ to $50 \%$ attempt suicide at some point of their life and another study reports that approximately $60 \%$ of all suicides occur in relation to mood disorders (15-16). 
With its chronic relapsing course BAD can be a severely disabling condition (17). In fact, bipolar disorder is the sixth leading cause of disability worldwide (18). BAD is associated with significant impairment in work, family and social functioning (19). Most affected individuals report a profoundly negative effect upon their quality of life, due to the illness (20).

Literature on the long-term course of this disorder in Asian populations is sparse. Some suggest that as for schizophrenia, the outcome for BAD is better in Asian countries, due to positive involvement of the family in these regions (21). The objective of this study was to describe the long-term course of BAD in patients in two general hospital clinic settings, in Sri Lanka, based on data extracted from clinic records.

\section{Methodology}

This was a descriptive cross sectional study. It was carried out at outpatient psychiatric clinics of Colombo North Teaching Hospital, Ragama and General (Teaching) Hospital, Kegalle, Sri Lanka.

The eligible study population comprised of patients from both genders aged 18-85 years, who were diagnosed to have BAD. The diagnosis had been made by a consultant psychiatrist, by clinical interview, based on the International Classification of Diseases version 10 (ICD 10) criteria. Those who had experienced the illness for less than one year, and those who were suffering from acute exacerbations, schizoaffective disorder or alcohol or drug misuse were excluded.

All eligible individuals were invited to participate and included if they gave informed consent. The recruitment process was continued until the pre-planned sample size of 350 participants was reached. Ethical clearance for the study was obtained from the Ethics Review Committee of the Faculty of Medicine, University of Kelaniya, Sri Lanka.

Data was collected from the patients, carers and from their clinic records using a pre-tested interviewer administered questionnaire, which examined for socio demographic characteristics, duration of the illness, number of relapses, treatment adherence, a past history of suicidal behaviour and functional level.

Statistical analysis was carried out using statistical Package for Social Science (SPSS) version 17.0 software program and Chi-square test was administered for categorical data.

\section{Results}

\section{Demographic details}

There were 350 participants, of whom 55.7\% (n=195) were female (Table 1). The age range of participants was from 18 to 85 years. Most (41.7\%) were aged between 41 to 55 years.

A majority of participants were married (62\%), most (62.6\%) were living with their spouse or children at the time of the study, and most (66.9\%) were unemployed. A majority had differing levels of formal education, and only $1.4 \%$ had never attended school.

\section{The course of BAD in relation to illness duration}

One third of the study population (32.6\%) had experienced the illness for 6-10 years and one fourth (24.6\%) for more than 20 years. Most (43.4\%) developed their illness before the age of 25 years, while $29.7 \%$ had the onset between 26 to 40 years, and the remaining $26.9 \%$ had the onset after 40 years.

The mean age at onset of the illness in the study sample was 31.7 years; 31.3 years for males and 31.9 years for females. Of the 155 male participants in this study sample, $45.2 \%(n=70)$ developed their illness before 25 years and 28.4\% ( $n=44)$ had the onset after 25 years but before 40 years. Among females, 42.6\% $(n=83)$ experienced onset before 25 years, and $30.3 \%(n=59)$ between $26-40$ years of age. There was no association between gender and age at onset.

Amongst a majority (70.0\%), mania was recorded as the first episode of illness, and in $25.7 \%$ depression was the first episode. Most (65.6\%) who had depression as the first episode were females. Only $34.4 \%$ of males were recorded to have experienced depression as the first episode of their illness. There was a significant association between type of first episode and gender $\left(\chi^{2}=6.086, p=0.048\right)$. Among patients who had mixed episodes, 33.3\% $(n=5)$ were males and $66.6 \%(n=10)$ were females.

Among the participants $37.4 \%(n=132)$, had a positive family history of mental illness. More than half (50.7\%) of the patients with a positive family history had the onset of illness at a younger age $\left(\chi^{2}=12.435, \mathrm{p}=0.014\right)$.

\section{The course of BAD in relation to relapses Overall patterns of relapse}

A majority (66\%) of participants had experienced more than one relapse by the time of the interview. Most (43.2\%) had 2-5 relapses, $11.4 \%$ had 6-10 relapses, $7.7 \%$ had more than 10 but less than 15 relapses, and 3.7\% had more than 20 relapses.

\section{First relapse}

Most patients (54.9\%) had experienced their first relapse within 2-5 years of onset of their illness. Of these, $27.7 \%$ had their first relapse within one year. The first relapse occurred in $6-10$ years in $8.9 \%$, after 10 years in $6.3 \%$ and after 20 years in $2.3 \%$. 


\section{Second relapse}

One third (33.4\%) of patients had no second relapse. But $38.3 \%$ relapsed for the second time within $2-5$ years of their illness. Another $15.1 \%$ experienced their second relapse within $6-10$ years and $8.3 \%$ relapsed after 11 years but before 20 years. A minority (2.3\%) had a second relapse within the first year and an almost similar number (2.6\%) had the second relapse after 20 years of their illness.

The likelihood of a relapse for each patient per year was 0.644 in the first 5 years, 0.405 in the next 5 - 10 years, 0.38 in the next $10-15$ years and 0.30 after 15 years. The trend was suggestive of fewer episodes over time.

\section{Types of relapse and associated factors}

Nearly half (49.1\%) experienced manic episodes most often, during relapses, while $15.1 \%$ had more depressive episodes. Of the remainder, (31.4\%), experienced both manic and depressive relapses equally and a small number (4.3\%) had mixed episodes.

In a majority (58.0\%), medication noncompliance was the main reported reason for relapse. Life stresses (29.1\%) were the next common reason for relapse (Table 1).

\begin{tabular}{|lrc|}
\hline $\begin{array}{l}\text { Table 1. Reasons for relapse among study } \\
\text { participants with BAD }\end{array}$ & No & Percentage \\
\hline Reason for relapse & 203 & $58.0 \%$ \\
\hline Defaulted medication & 102 & $29.1 \%$ \\
Life stresses & 12 & $3.4 \%$ \\
Defaulted medication + stresses & 9 & $2.6 \%$ \\
Treatment resistance & 15 & $4.3 \%$ \\
Other causes & 9 & $2.6 \%$ \\
More than one cause & 350 & 100.0 \\
Total & & \\
\hline
\end{tabular}

\section{The course of BAD in relation to treatment adherence}

A majority of participants (62\%) in this study reported good drug compliance at the time of interview. Poor treatment compliance was significantly associated with the number of relapses $\left(\chi^{2}=92.992, p=0.000\right)$. Many participants (59.7\%) expressed the belief that they would relapse if they stopped medication. But $23.7 \%$ believed that they would not relapse if they stopped medication.

Some (12.3\%) were worried about side effects of medication and a minority (4.6\%) had no knowledge about their medication. A significantly higher relapse rate was seen in those with negative ideas about medication $\left(\chi^{2}=69.213\right.$, $\left.\mathrm{p}=0.000\right)$.
A large majority (83.4\%) were happy about the support given by their families to recover from their illness. However $7.4 \%$ had poor family support.

\section{The course of BAD in relation to suicidal attempts}

Nearly one fifth (18.9\%) of patients had a past history of a suicidal attempt at the time of the interview, of whom two thirds were females $(68.2 \%)\left(\chi^{2}=7.607\right.$, $\mathrm{p}=0.022$ ).

When considering the methods, $51.5 \%$ tried to harm themselves by jumping in to a well, river or from a height (Table 2).

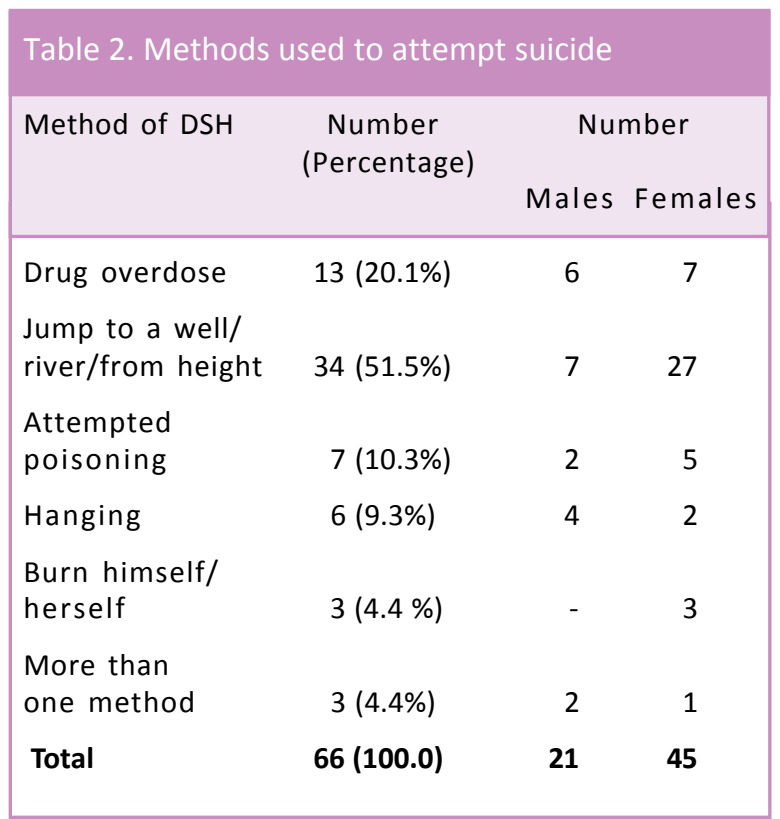

\section{Functional outcome}

The majority (60.3\%) had been reportedly functioning well (studying or employed) at the time of onset of BAD. At the time of the interview, only $33.1 \%(n=116)$ were functioning well.

\section{Discussion}

The findings of this naturalistic study, conducted in a large teaching hospital clinic, shows that patients with BAD experience a diverse course of their illness, with time. In this study population, the number of relapses appeared to reduce with time. Other studies too show that evidence-based long-term treatment with regular follow-up visits, could improve the course of the disease and the prognosis (22). Treatment compliance was associated with fewer relapses in this study.

Relapses were common, with more than half having their first relapse within 5 years and a quarter within one year. 
However, a minority had their second relapse 20 years after the first episode. Predicting the chance of relapse in individuals therefore remains elusive. Another study done in Chicago too showed that more than half of their study population were re-hospitalised due to a relapse at least once in 4-5 years after diagnosis (17).

The mean age of onset of participants in this study appears to be late, in marked contrast to the accepted global mean age of onset of 21 years for BAD (23). This is perhaps due to recall bias, which is a major limitation in retrospective studies. Alternatively, participants may not have presented to services during the initial episodes of the illness - the early episodes may have been missed, with spontaneous remission, particularly if they were depressive or hypomanic in nature. This may also be a reason why males presented with initial manic episodes, in contrast to females who presented with depressive episodes. Males are often less likely to seek help for depressive episodes compared to females, and therefore may not present to the services early until a manic episode necessitates help. It is noteworthy that another study from USA too reports that bipolar females were more likely to have a depressive onset of BAD (24).

Half of those with BAD in our study most often experienced manic relapses, nearly one third had mania and depression equally, and only a minority experienced mostly depressive relapses. This difference again could be due to 'unnoticed' depressive episodes, as seen at the onset of illness.

Reinforcing the fact that BAD has strong genetic and biological underpinnings, our study showed that more than one third (37.7\%) of participants have a positive family history of mental illness, and more than half with a positive family history developed their illness before the age of 25 years $(7,8,25)$.

A majority of patients in this study had good treatment adherence at the time of interview. A study from UK reports a similar percentage (40\%) of medication non adherence (36). Many study participants believed that they would relapse if they discontinued the medication. There was a clear association between the patients' idea about the treatment and number of relapses. Patients with negative ideas about treatment had a higher relapse rate.

One fifth of patients with BAD had attempted suicide in our study. Other studies have reported higher figures, with differing studies reporting life-time prevalence of suicidal attempts in people with BAD to be $50 \%$ (27), between $25-50 \%$ (4) and 33\% in southern Spain (16). It is worth exploring whether the greater family support in middle-income countries such as ours is a reason for the reported fewer suicidal attempts. It is possible that other factors, such as recall bias, and a reticence to reveal such information, may also have influenced our findings.
More than two thirds of patients who attempted suicide were females, which is in keeping with patterns of attempted suicide and self-harm in the general population (28). Previous work on BAD from Barcelona too showed females to have higher levels of self-harm, compared to males (4).

BAD was associated with an apparent functional decline among participants in our study, with the numbers studying or employed at the time of onset of the disorder declining to half by the time of the interview. The Harvard Health Publications (June 2008) reports that $57 \%$ of persons with $\mathrm{BAD}$ were unable to work at all due to their disorder (29). Functional impairment could also be due to untreated depressive episodes. In this study it is difficult to conclude this functioning drop was due to BAD alone, as our study population included participants from 15 to 85 years of age, who may have showed natural progression and retirement.

\section{Limitations}

This study is limited in its scope as it relies heavily on clinic records, which may have gaps in documentation. Furthermore patients in Sri Lanka may change their doctors or clinics during relapses and get admitted to other hospitals other than their primary clinic during relapses. Such relapses would have been missed in this survey. Patients with substance use disorders were excluded, which may also have influenced findings.

\section{Conclusions}

Despite these limitations, this study documents the naturalistic trends of persons with BAD in Sri Lanka, which would be useful for understanding longer-term outcomes, and planning services, for patients with BAD attending hospital clinics in this country.

\section{Disclosure statement}

None declared

P Kandapola Arachchige, General Hospital, Gampaha

KMIWM Senevirathne, VP Eranga, MUPK Peris, KALA Kuruppuarachchi, SS Williams, Department of Psychiatry, Faculty of Medicine, University of Kelaniya

PLN Fernando, Colombo North Teaching Hospital, Ragama

Corresponding author: SS Williams

Email: shehan.williams@linacre-oxford.com

https://orcid.org/0000-0001-7764-5550 


\section{References}

1. Sadock BJ, Sadock VA. Kaplan and Sadock's Synopsis of Psychiatry. 10th ed. Philadelphia: Lippincot Williams and Wilkins, 2007.

2. World Health Organization (WHO). The ICD 10 Classification of mental, behavioural disorders; Clinical description and diagnostic guidelines. Geneva: WHO, 1992.

3. Christensen EM, Gierris A, Larsen JK, et al. Life events and onset of a new phase in Bipolar Affective Disorder. Bipolar Disord 2003; 5(5): 356-61.

4. Nivoli AM, Pucchiarotti I, Rosa AR, et al. Gender difference in a cohort study of 604 Bipolar patients: the role of predominant polarity. J Affect Disord 2011;133(3): 443-9.

5. Cowen P, Harrison P, Burns T. Shorter Oxford Textbook of Psychiatry. 6th ed. Oxford: Oxford University Press, 2012.

6. Manczor M. Bipolar Disorders in the elderly. Vertex 2010; 21(92): 275-83.

7. Craddock N, Jores I. Genetics of bipolar disorder. J Med Genet 1999; 36: 585-594.

8. Edvardsen J, Torgersen S, Roysamb E, et al. Heritability of bipolar spectrum disorders: Unity or heterogenicity. J Affect Disord 2008; 106(3): 229-40.

9. Serritti A, Mandelli L. The genesis of bipolar disorder: genome 'hot regions' genes, new potential candidates and future directions. Mol Psychiatry 2008; 13(8): 742-71.

10. Alloy LB, Abramson LY, Urosevie S, et al .The psychosocial context of bipolar disorder: environmental, cognitive and developmental risk factors. Clin Psychol Rev 2005; 25(8): 1043-75.

11. Chengappa KN, Kupfer DJ, Frank E, et al. Relationship of birth cohort and early age at onset of illness in a bipolar disorder case registry. Am J Psychiatry 2003;160: 1636-42.

12. Colom F, Vieta E, Martinez-Aran A, et al. Clinical features associated with treatment non compliance with bipolar disorder. J Clin Psychiatry 2000; 61(8): 549-55.

13. Silverstone T, Romans CS. Bipolar affective disorder: causes and prevention of relapses. Br J Psychiatry 1989;154:321-35.

14. Barraclough B, Bunch J, Nelson B, et al. One hundred cases of suicide: clinical aspects. Br J Psychiatry 1974; 125: $355-73$

15. Novick DM, Swartz HA, Frank E. Suicide attempts in Bipolar 1 and 11 disease: A review and meta analysis of the evidence. Bipolar disord 2010; 12(1): 1-9.
16. Lopez P, Mosquera F, De Leon J, et al. Suicide attempts in Bipolar Patients. J Clin Psychiatry 2001; 62(12): 963-6.

17. Goldberg J E, Harrow M, Grossman LS. Course and outcome in bipolar affective disorder: A longitudinal follow up study. Am J Psychiatry 1995; 152(3): 379-84.

18. Mathers C, Boerma JT, Fat DM. The global burden of diseases: 2004 Update. Geneva; World Health Organization, 2008.

19. Sanchez-Moreno J, Martinez-Aran A, Tabares-Seisdedos $\mathrm{R}$, et al. Functioning and disability in bipolar disorder: An extensive review. Psychother Psychosom 2009; 78(5): 285-97.

20. Michalak EE, Murray, Young AH, et al. Burden of bipolar depression-impact of disorder and medication on quality of life. CNS Drugs 2008; 22(5): 389-406.

21. Hopper K, Wanderling J. Revisiting the Developed vs Developing country distinction in course and outcome in Schizophrenia: Results from ISoS, the WHO collaborative follow up project. International Study of Schizophrenia. Schizophr Bull 2000; 26(4): 835-46.

22. Tundo A, Musetti L, Benedetti A, et al. Predictors of recurrence during long-term treatment of bipolar I and II disorders. A 4 year prospective naturalistic study. J Affect Disord 2017; 225: 123-8.

23. Weissman MM, Blend RC, Canino GJ, et al. Cross National epidemiology of major depression and bipolar disorder. JAMA 1996; 276(4): 293-9.

24. Altshuler LL, Kupka RW, Hellemann G, et al. Gender with depressive symptoms in 711 patients with Bipolar Disorder evaluated prospectively in the Stanley Foundation bipolar treatment outcome network. Am J Psychiatry 2010; 167(6): 708-15.

25. Kato T. Molecular genetics of bipolar disorder and depression. Psychiatry Clin Neurosci 2007; 6(1): 3-19.

26. Lingam $\mathrm{R}$, Scott J. Treatment non adherence in affective disorders. Acta Psychiatr 2002;105(3):164-72.

27. Morris R, Maeshall M, Harris A. Bipolar affective disorder left out in the cold: too late for the national service framework but local initiative may be possible. BMJ 2002; 324: 61-2.

28. Muller A, Claes L, Smits D, et al. Prevalence and Correlates of self harm in the German General Population. PLoS ONE 2016; 11(6): e0157928.

29. Harvard Mental Health Letter. Improving outcomes in bipolar disorder. Boston: Harvard Health Publishing, 2008. Available from: https://www.health.harvard.edu/newsletter_article/ Improving_outcomes_in_bipolar_disorder 